\title{
Prospective longitudinal quality of life and survival outcomes in patients with advanced infiltrative hepatocellular carcinoma and portal vein thrombosis treated with Yttrium-90 radioembolization
}

\author{
Minzhi Xing ${ }^{1}$, Nima Kokabi ${ }^{1}$, Juan C. Camacho ${ }^{2}$ and Hyun S. Kim ${ }^{1,3^{*}}$
}

\begin{abstract}
Background: To determine the effect of Yttrium-90 (Y90) radioembolization on health-related quality of life (HRQOL) and its effect on overall survival advanced, unresectable infiltrative hepatocellular carcinoma (HCC) patients with concurrent portal vein thrombosis (PVT).

Methods: Consecutive patients with unresectable infiltrative HCC and PVT were recruited. The Short-Form 36 (SF-36) questionnaire was used to assess HRQOL for consecutive patients treated with glass-based Y90 based on a prospective phase II trial. MR imaging was used to determine tumor progression every 3 months post-treatment. Overall survival (OS) from treatment and time to progression (TTP) was analyzed using Kaplan-Meier estimation and log-rank test.
\end{abstract}

Results: Thirty patients were treated and followed for 17.4 months; physical and mental component summary scores (PCS \& MCS) remained unchanged at one, three, and six months. While no difference was observed in baseline SF-36 scores for patients with prolonged TTP ( $\geq 4$ months) and OS ( $\geq 6$ months), corresponding 1-month PCS were significantly higher than those with TTP $<4$ months and OS $<6$ months. At 1 month, patients with normalized Physical Function (PF), Role Physical (RP) and PCS within 2 standard deviations (SD) of US normalized baseline scores had a significantly prolonged median OS (15.7 vs. 3.7 months; $p<0.001)$ and TTP (12.4 vs. 1.8 mo; $p<0.001)$ compared those with physical component scores greater than 2SD below normalized US population values.

Conclusion: Y90 radioembolization for HCC demonstrated long-term preservation of HRQOL. Lower baseline HRQOL scores were predictive of poorer OS. Early (1 month post-treatment) significant decreases in PCS were independent predictors of poorer OS and TTP.

Trial registration: ClinicalTrials.gov identifier NCT01556282, registered March 16, 2012.

Keywords: Hepatocellular carcinoma, Quality of life, Yttrium-90 radioembolization, Locoregional therapies

\footnotetext{
* Correspondence: kevin.kim@yale.edu

'Interventional Radiology, Department of Radiology and Biomedical Imaging,

Yale University School of Medicine, New Haven, CT, USA

${ }^{3}$ Yale Cancer Center, Yale University School of Medicine, 330 Cedar Street, TE

2-224, New Haven, CT 06510, USA

Full list of author information is available at the end of the article
} 


\section{Background}

Worldwide, hepatocellular carcinoma (HCC) ranks as the sixth most prevalent cancer and causes the third most cancer-related deaths [1-3]. At diagnosis, approximately 10 to $40 \%$ of $\mathrm{HCC}$ patients have portal vein thrombosis concurrently $[4,5]$. These patients often have very poor prognosis, and are not eligible for curative surgery via resection or transplantation. Without treatment, the reported median overall survival (OS) for Barcelona Clinic Liver Cancer (BCLC) Stage $\mathrm{C}$ patients range from 2 months to 4.8 months, as compared to 10 to 24 months in HCC patients without PVT [5-9].

Due to the risk of hepatic artery infarction, embolic intra-arterial therapies such as transarterial chemoembolization (TACE) have previously been deemed as contraindications in HCC with PVT $[10,11]$. However, more recent studies establishing the safety and efficacy of conventional TACE (CTACE) in prolonging survival for HCC patients with PVT have been conducted [12, 13]. Nevertheless, increased survival benefits have not been specifically established in infiltrative HCC patients [14]. In long-term studies, selective intra-arterial yttrium$90\left({ }^{90} \mathrm{Y}\right)$ radioembolization has demonstrated equal or better overall survival (OS) and time to progression (TTP) vs. cTACE [15], with comparable safety and efficacy in patients with infiltrative HCC with PVT [16]. Given the lowered risk of hepatic ischemia and decreased embolic effect with ${ }^{90} \mathrm{Y}$ radioembolization compared to cTACE, some authors have noted that it may be a preferred therapeutic option for patients with concurrent PVT [10, 12, 13].

In palliative oncology, health-related quality of life (HRQOL) has become an increasingly relevant measure of patient prognosis. In recent years, a progressively higher number of investigators have suggested using HRQOL as an independent prognostic factor for response to treatment and progression of disease in patients with advanced HCC [17, 18]. As a widely-used tool in the evaluation of HRQOL, the Short-Form 36 (SF-36) contains 36 items/8 domains, including physical and social well-being, vitality, and pain [19]. In HCC patients, significant improvements in mental health at four months post-therapy [20] have been reported using the SF-36 for patients treated with cTACE, while significant increases in HRQOL with ${ }^{90} \mathrm{Y}$ radioembolization have been observed at one month following therapy [21]. However, to date no studies have demonstrated both survival outcomes of ${ }^{90} \mathrm{Y}$ therapy and its effect on HRQOL in unresectable, infiltrative HCC with PVT in particular. Our purpose was to investigate the effect of ${ }^{90} \mathrm{Y}$ radioembolization on HRQOL in patients with infiltrative, unresectable $\mathrm{HCC}$ with PVT, and its effect on overall survival (OS) and time to progression (TTP). We hypothesized that patients with higher HRQOL scores prior to therapy would demonstrate more favorable survival outcomes, with maintenance of HRQOL posttherapy.

\section{Methods \\ Study design and patient selection}

In an Institutional Review Board (IRB) approved correlative study based on a prospective phase II trial (trial registration at ClinicalTrials.gov; identifier NCT01556282, registered March 16, 2012), consecutive patients with infiltrative HCC with PVT were enrolled for possible therapy with glass-based ${ }^{90} \mathrm{Y}$ radioembolization. Diagnosis of HCC was made according to American Association for Study of the Liver Diseases (AASLD) using dynamic contrast-enhanced magnetic resonance imaging (MRI). All patients in this study had PVT due to tumor invasion. As previously described, infiltrative $\mathrm{HCC}$ was defined as a geographic region with high T2 signal, arterial enhancement and early washout on $\mathrm{T} 1$ gadolinium-enhanced images [22]. A multidisciplinary liver cancer tumor board evaluated every patient for his/her suitably to undergo ${ }^{90} \mathrm{Y}$ radioembolization.

\section{Pre-treatment evaluation, planning, inclusion/exclusion criteria, and ${ }^{90} \mathrm{Y}$ therapy}

As previously described by our group, patients who were $\geq 18$ years with Eastern Cooperative Oncology Group (ECOG) performance score (PS) $\leq 2$ and life expectancy of at least 3 months were included. Additionally, those with tumor burden $<75 \%$ of entire liver, and adequate liver reserve based on liver function tests (serum bilirubin $<2 \mathrm{mg} / \mathrm{dl}$, serum albumin $>2.5 \mathrm{~g} / \mathrm{dl}$, and/or AST or ALT $<5$ times the upper limit of normal) were included. Following lung shunt study, those with estimated lung dose >30Gy, lung shunt fraction (LSF) $>20 \%$, observable uncorrectable GI flow on diagnostic angiogram were excluded from this study as previously described by the authors [23]. In general, 120 Gy dosimetry to the treated lobe of the liver was planned based on Therasphere $^{\oplus}$ (Biocampatibles, London, UK) published guidelines $[12,23]$.

\section{Clinical follow-up and health-related quality of life assessment}

Patients were followed up in the Interventional Oncology Clinic at one week post-therapy, and subsequently at one month, three months, and every three months thereafter until death. The SF-36 Health Survey Form (version 1) assessment tool was used to measure HRQOL scores at the initial pre-treatment visit and at each subsequent posttherapy 1-, 3- and 6-month follow-up visit, with a conversion of raw scores to a $0-100$ scale [19]. In line with the SF-36 Health Survey Manual and Interpretation Guide [17], the survey was self-administered by patients and scored independently. 


\section{Statistical analysis}

Overall mean scores were compared at each time point. HRQOL scores of each domain pre- and post-therapy were compared using paired t-tests. The chi-squared $\left(x^{2}\right)$ test was used to compare categorical variables. Using the Kaplan-Meier method overall survival (OS) times were calculated from initial ${ }^{90} \mathrm{Y}$ procedure. SAS version 9.3 (SAS Institute Inc., Cary, NC, USA) software was used for all computations.

\section{Results}

\section{Overall baseline characteristics}

Between 2011 and 2013, thirty patients with advanced, infiltrative $\mathrm{HCC}$ with $\mathrm{PVT}$ received glass-based ${ }^{90} \mathrm{Y}$ radioembolization. All patients in this study had PVT due to tumor invasion. Detailed patient demographics, tumor morphology, and characteristics of portal vein thrombosis at initial presentation are shown in Table 1. Median age at baseline was 62 years (range 35-92), with the majority being male $(n=23 ; 77 \%)$ and of Caucasian ethnicity $(n=24 ; 80 \%)$. At baseline, 20 patients $(67 \%)$ had documented Child Pugh A disease, and the majority had an Eastern Co-operative Oncology Group (ECOG) performance score of $1(n=17 ; 57 \%)$. All 30 patients demonstrated PVT on MR imaging and were thus classified as BCLC Stage C, with most patients demonstrating occlusive PVT $(n=23 ; 77 \%)$ occurring in a branch of the portal vein $(n=24,80 \%)$. Twenty-one patients $(70 \%)$ had evidence of hepatic cirrhosis and/or portal hypertension on baseline MR imaging. Twenty-two patients (73\%) had a non-liver-related comorbidity at the baseline, and the majority of these patients suffered from hypertension and/ or diabetes. Overall at the time of receipt of ${ }^{90} \mathrm{Y}$ radioembolization, there were 6 patients who were receiving Sorafenib concurrently, and an additional 11 patients had received Sorafenib prior to ${ }^{90} \mathrm{Y}$ therapy. With the exception of Sorafenib, no other concurrent locoregional or systemic HCC-directed therapy was received.

\section{SF-36 survey results}

Of the 30 patients, all patients completed 1-month follow-up surveys, with 26 (87\%) and 20 (67\%) having completed 3-month and 6-month follow-up surveys respectively at time of data collection. The decline in response rate at was due to 4 patient deaths at 3 months and 10 patients deaths by 6 months. Twenty-three patients $(77 \%)$ were deceased at the time of data analysis. The median time interval after initial ${ }^{90} \mathrm{Y}$ radioembolization therapy to the first follow-up survey was 4.5 weeks. Median follow-up time for all patients was 17.4 months following initial ${ }^{90} \mathrm{Y}$ therapy. Lower baseline raw SF-36 scores in each of the 8 domains were observed on comparison to the raw scores of a healthy, age-adjusted healthy population in the US (Fig. 1a).
Additionally, the mean SF-36 HRQOL Normalized Baseline Scores (NBS) in all domains were significantly lower when compared to mean scores of the healthy, age-adjusted population in the US $(p<0.001)$ (Fig. 1b). Baseline physical component scores, including Physical Functioning (PF) and Role-Physical (RP), as well as the overall Physical Component Summary Score (PCS) were more substantially decreased vs. age-adjusted norms compared to mental component scores (Fig. 1b).

\section{HRQOL analysis}

There were no significant changes in any of the 8 domains at 1 -month, 3 -month and 6 -months post $-{ }^{90} \mathrm{Y}$ therapy compared to pre- ${ }^{90} \mathrm{Y}$ baseline scores on longitudinal analysis (Fig. 2). When comparing scores at 1-month vs. 3 months post $-{ }^{90} \mathrm{Y}$ therapy, there were non-statistically significant positive trends in Role-Physical (20.37 vs. 22.06, $p=0.27$ ) and Vitality (31.85 vs. 35.22, $p=0.11$ ) domains; however, a significant increase in the RoleEmotional score (53.09 vs. 60.78, $p=0.04$ ) was observed. At 3 months post- ${ }^{90} \mathrm{Y}$ therapy, a negative trend in the General Health domain (44.09 vs. 41.52, $p=0.07$ ) almost reached significance. Longitudinal analysis of all 8 domains (Fig. 2) demonstrated no significant changes between follow-up in the short-term within 1-3 months vs. 6-month scores. A comparison of 3-month vs. 6month follow-up scores revealed a nonsignificant decrease in the Role-Emotional domain (60.78 vs. 48.48, $p=0.06)$ and an increase in the Bodily Pain domain (51.62 vs. $57.95, p=0.08$ ). No significant changes in either the physical component summary scores (PCS) or mental component summary scores (MCS) were observed at all follow-up intervals when compared to baseline, or between 1-vs. 3-month or 3-vs. 6-month interval scores $(p>0.05$ for all).

\section{${ }^{90} \mathrm{Y}$ Radioembolization and physical component summary scores}

Baseline characteristics were further stratified based on PCS at 1 -month post- ${ }^{90} \mathrm{Y}$ therapy (Table 1). Eighteen patients (60\%) had 1-month PCS within 2 standard deviations (SD) of US age-adjusted healthy normalized baseline scores, while 12 patients (40\%) had 1-month PCS greater than 2 SD below that of US age-adjusted healthy NBS. No significant differences in baseline characteristics were found between patients who had 1month post $-{ }^{90} \mathrm{Y}$ PCS within $2 \mathrm{SD}$ vs. $>2$ SD below that of mean NBS. However, it was noted that baseline mean largest tumor size was significantly greater in patients with 1-month PCS within 2SD of NBS $(9.7 \mathrm{~cm}$ vs. $9.1 \mathrm{~cm}, p=0.04)$. 
Table 1 Patient baseline demographics and clinical characteristics at enrollment $(n=30)$

\begin{tabular}{|c|c|c|c|c|c|c|}
\hline \multicolumn{2}{|l|}{ Patient Characteristics } & Parameters & Overall n (\%) & $\begin{array}{l}1 \text { month PCS within } \\
2 \text { SD of NBS }(n=18)\end{array}$ & $\begin{array}{l}1 \text { month PCS > 2SD } \\
\text { below NBS }(n=12)\end{array}$ & $p$-value \\
\hline \multicolumn{2}{|l|}{$\overline{\text { Age }}$} & Median (range) & $62(35-82)$ & 60.8 & 61.3 & 0.46 \\
\hline \multirow{2}{*}{\multicolumn{2}{|c|}{ Gender }} & Male & $23(77 \%)$ & 13 & 10 & 0.31 \\
\hline & & Female & $7(23 \%)$ & 5 & 2 & \\
\hline \multirow{3}{*}{\multicolumn{2}{|c|}{ Ethnicity }} & White & $24(80 \%)$ & 14 & 10 & 0.37 \\
\hline & & Black & $4(13 \%)$ & 3 & 1 & \\
\hline & & Other & $2(7 \%)$ & 1 & 1 & \\
\hline \multirow{4}{*}{\multicolumn{2}{|c|}{ HCC Etiology }} & $\mathrm{HCV}$ & $16(53 \%)$ & 10 & 6 & 0.42 \\
\hline & & HBV & $2(7 \%)$ & 1 & 1 & \\
\hline & & ALD & $5(17 \%)$ & 3 & 2 & \\
\hline & & Other causes of cirrhosis & $7(23 \%)$ & 4 & 3 & \\
\hline \multirow{2}{*}{\multicolumn{2}{|c|}{ Non-Liver Comorbidities (e.g. Hypertension, Diabetes) }} & Present & $22(73 \%)$ & 14 & 8 & 0.25 \\
\hline & & Absent & $8(27 \%)$ & 4 & 4 & \\
\hline \multirow{2}{*}{\multicolumn{2}{|c|}{ Portal Hypertension }} & Present & $20(67 \%)$ & 11 & 9 & 0.43 \\
\hline & & Absent & $10(33 \%)$ & 7 & 3 & \\
\hline \multirow{2}{*}{\multicolumn{2}{|c|}{ Hepatic Encephalopathy }} & Present & $3(10 \%)$ & 1 & 2 & 0.33 \\
\hline & & Absent & $27(90 \%)$ & 17 & 10 & \\
\hline \multirow{3}{*}{\multicolumn{2}{|c|}{ Child-Pugh Class }} & A & $20(67 \%)$ & 11 & 9 & 0.19 \\
\hline & & B & $10(33 \%)$ & 7 & 3 & \\
\hline & & c & $0(0 \%)$ & 0 & 0 & \\
\hline \multirow{2}{*}{\multicolumn{2}{|c|}{ ECOG Performance Status }} & 0 & $13(43 \%)$ & 9 & 4 & 0.17 \\
\hline & & 1 & $17(57 \%)$ & 9 & 8 & \\
\hline \multirow[t]{9}{*}{ Tumor Morphology } & Tumor Locations & Unilobar & $19(63 \%)$ & 12 & 7 & 0.82 \\
\hline & & Bilobar & $11(37 \%)$ & 6 & 5 & \\
\hline & Number of Nodules & Solitary & $11(37 \%)$ & 7 & 4 & 0.23 \\
\hline & & Multiple & $19(63 \%)$ & 11 & 8 & \\
\hline & Mean largest tumor si & cm) & $9.2(4.9-19)$ & 9.7 & 9.1 & 0.04 \\
\hline & Mean largest tumor $\mathrm{v}$ & ne $\left(\mathrm{cm}^{3}\right)$ & $588(145-1136)$ & 580 & 602 & 0.42 \\
\hline & Tumor Burden & $<50 \%$ & $20(67 \%)$ & 11 & 9 & 0.20 \\
\hline & & $50 \%-75 \%$ & $10(33 \%)$ & 7 & 3 & \\
\hline & & $>75 \%$ & $0(0 \%)$ & 0 & 0 & \\
\hline \multirow[t]{6}{*}{ Portal Vein Thrombosis (PVT) } & Presence & Present & $30(100 \%)$ & 18 & 12 & 0.26 \\
\hline & & Absent & $0(0 \%)$ & 0 & 0 & \\
\hline & Location & Main PV & $6(20 \%)$ & 4 & 2 & 0.63 \\
\hline & & Branch PV & $24(80 \%)$ & 14 & 10 & \\
\hline & Degree of Occlusion & Occlusive & $23(77 \%)$ & 13 & 10 & 0.30 \\
\hline & & Non-Occlusive & $7(23 \%)$ & 5 & 2 & \\
\hline Laboratory Data & Mean Serum AFP (ng/ & & 715 (3.3 to >2400) & 703 & 801 & 0.36 \\
\hline \multirow[t]{2}{*}{ Previous Treatment } & & No & $10(33 \%)$ & 7 & 3 & 0.22 \\
\hline & & Yes & $20(67 \%)$ & 11 & 9 & \\
\hline \multirow{4}{*}{\multicolumn{2}{|c|}{ Type of Previous Treatment ${ }^{\mathrm{a}}$}} & cTACE/DEB-TACE & $14(47 \%)$ & 10 & 4 & 0.19 \\
\hline & & RFA & $0(0 \%)$ & 0 & 0 & \\
\hline & & Liver Resection & $0(0 \%)$ & 0 & 0 & \\
\hline & & Sorafenib ${ }^{a}$ & $6(20 \%)$ & 4 & 2 & \\
\hline \multicolumn{2}{|l|}{ Lung Shunt Fraction (LSF) } & Mean (\%) & $11.0(5.1-19.8)$ & 11.3 & 10.8 & 0.52 \\
\hline
\end{tabular}

PCS Physical Component Summary Scores, SD Standard Deviation, ECOG Eastern Cooperative Oncology Group, PVT Portal Vein Thrombosis, cTACE Conventional Transarterial Chemoembolization, DEB-TACE Drug-Eluting Bead Transarterial Chemoembolization, RFA Radiofrequency Ablation ${ }^{a}$ Indicates number of patients who were taking Sorafenib at the time of ${ }^{90} \mathrm{Y}$. A total of 17 patients $(55 \%)$ had been on Sorafenib at the time of or prior to receiving ${ }^{90} \mathrm{Y}$ therapy

Bold data are statistically significant $(P<0.05)$ 

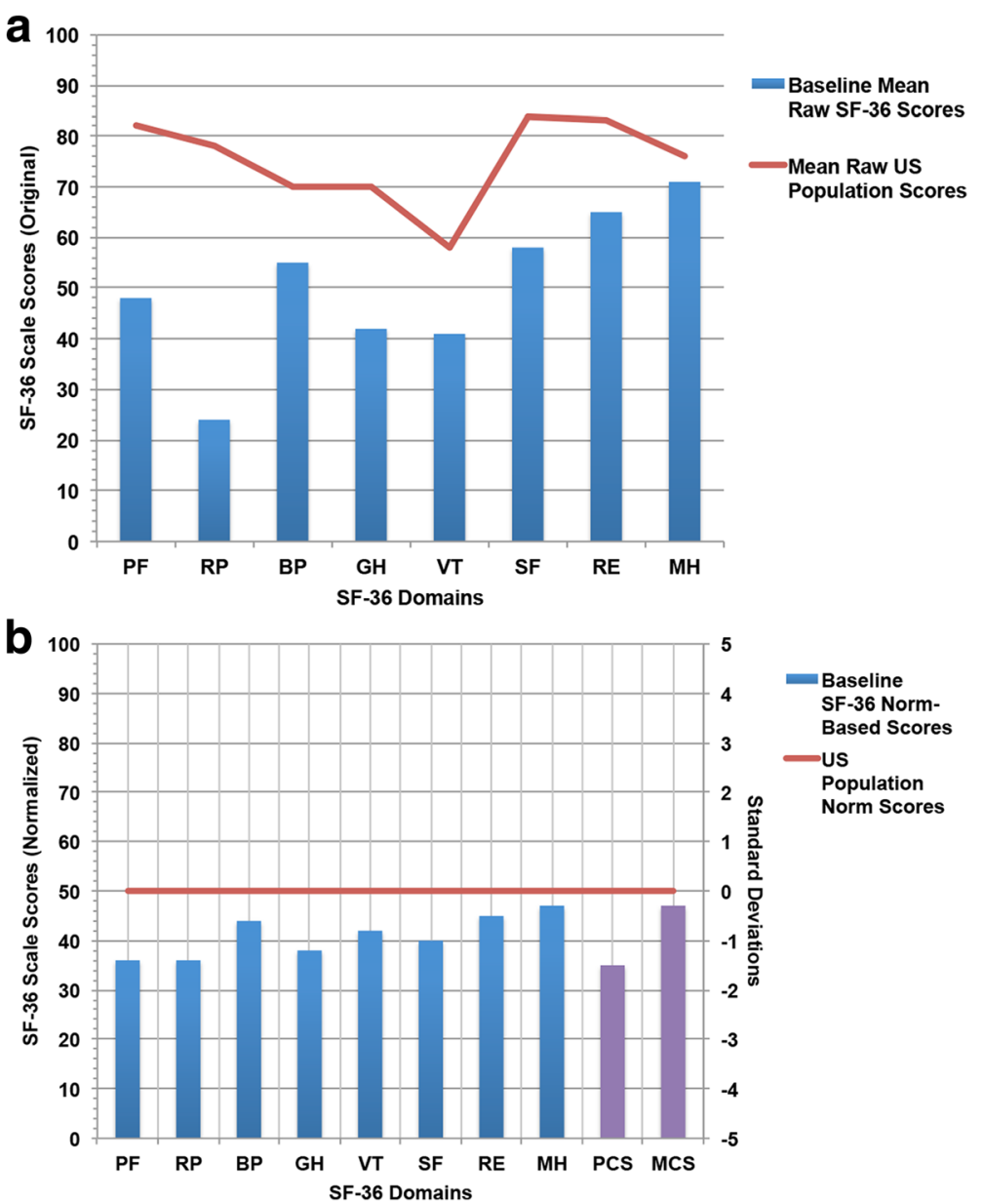

Fig. 1 Comparison of mean SF-36 HRQOL Normalized Baseline Scores for patients with HCC prior to Y90 therapy (bars) versus mean SF-36 HRQOL scores of an age-adjusted healthy US population (lines). a Raw SF-36 scores compared to US population means; (b) Normalized SF-36 scores. Significantly lower HRQOL scores were observed in patients at baseline compared to the mean US healthy population $(p<0.001)$. PF: Physical Functioning; RP: Role-Physical; BP: Bodily Pain; GH: General Health; VT: Vitality; SF: Social Functioning; RE: Role Emotional; MH: Mental Health; MCS: Mental Component Summary Score; PCS: Physical Component Summary Score

\section{Survival and outcomes}

The median OS from initial ${ }^{90} \mathrm{Y}$ radioembolization was 13.0 months (95\% confidence interval (CI), 4.4-22 months) while median overall time to progression was 9.0 months (95\% CI 6.2-13.1 months). Patients with PCS within 2SD of NBS demonstrated significantly prolonged median OS (15.7 months vs. 3.7 months, $p<0.001$; Fig. 3a) and significantly prolonged TTP (12.4 months vs. 1.8 months, $p<$ 0.001 ; Fig. 3b) when compared those with PCS $>2$ SD below the healthy, normalized US population.. Overall 3-month and 6-month survival rates from ${ }^{90} \mathrm{Y}$ therapy were $86.7 \%$ and $66.7 \%$, respectively. Three-month survival rates for patients with 1 month post- ${ }^{90} \mathrm{Y}$ PCS within 2SD was $94.4 \%$, compared to $83.3 \%$ for patients with 1 month post- ${ }^{90} \mathrm{Y}$ PCS $>2$ SD below NBS, $p=0.01$. Six-month survival rates were $72.2 \%$ vs. $58.3 \%$ for patients with 1 month post- ${ }^{90} \mathrm{Y}$ PCS within $2 \mathrm{SD}$ vs. $>2 \mathrm{SD}$ below NBS, $p=0.005$. On multivariate analysis, ECOG performance score (hazard ratio (HR)
11.29, $p=0.021$ ), Child Pugh class (HR 6.32, $p=0.013$ ), Lung Shunt fraction (HR 6.42, $p=0.029$ ), and PCS at 1month post $-{ }^{90} \mathrm{Y}$ therapy (HR 1.73, $p=0.041$ ) were significant independent predictors of overall survival. In addition, these same factors were also significant independent prognostic factors for time to progression (Table 2).

\section{Discussion}

This study examined the efficacy of ${ }^{90} \mathrm{Y}$ radioembolization therapy on HRQOL in patients with advanced, infiltrative $\mathrm{HCC}$ with PVT. Currently, ${ }^{90} \mathrm{Y}$ radioembolization is considered a viable therapeutic option for palliative treatment of advanced stage HCC patients who are not candidates for surgery. As our results demonstrated, such patients tended to have baseline HRQOL scores significantly below that of the normal age-adjusted healthy population. Given the overwhelmingly poor prognosis of infiltrative HCC with PVT and the tendency to have exhausted other 


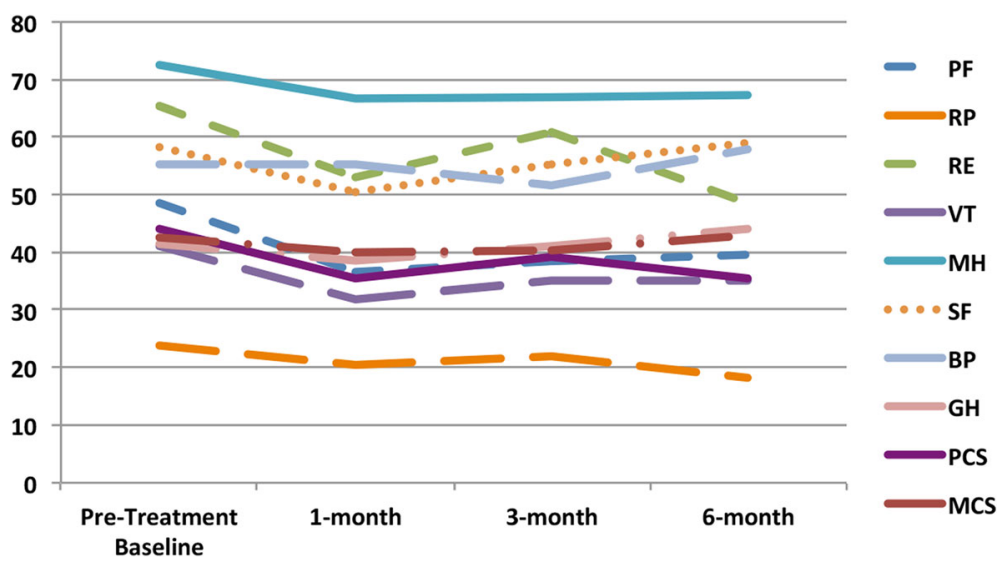

Fig. 2 Longitudinal analysis of HRQOL after Y90 radioembolization demonstrating lack of significant change in health-related quality of life pre- and post-Y90 therapy, and over the course of 6-month follow-up. PF: Physical Functioning; RP: Role-Physical; BP: Bodily Pain; GH: General Health; VT: Vitality; SF: Social Functioning; RE: Role Emotional; MH: Mental Health

therapeutic options in such patients, it is imperative to ensure that any treatment rendered does not further worsen patients' quality of life.

The survival benefits of ${ }^{90} \mathrm{Y}$ radioembolization therapy on HRQOL in infiltrative, advanced HCC with PVT have not been extensively characterized in the literature for adequate comparison with the current study. However, in a cohort of patients with majority intermediateadvanced (BCLC $\mathrm{B} / \mathrm{C}$ ) disease, ${ }^{90} \mathrm{Y}$ radioembolization therapy gave rise to significant increases in social and functional well-being at 1 month, without significant changes in overall HRQOL scores [21]. In addition, for HCC patients without PVT, ${ }^{90} \mathrm{Y}$ radioembolization therapy improved HRQOL scores compared to cisplatin administered through hepatic arterial infusion (HAI) [24], with significantly higher functional well being at 3- months and 6-months post $-{ }^{90} \mathrm{Y}$ therapy vs. cisplatin HAI. Previous studies investigating HRQOL patients receiving other forms of intra-arterial therapy such as transarterial embolization or CTACE have reported decreased HRQOL was at 3 month follow-up compared to baseline $[25,26]$, and improved HRQOL at 6 months vs. 3 months [25]. A study by Wible et al. [20] indicated that HCC patients who received $\geq 3$ cTACE procedures had significantly improved mental health scores following the initial procedure (13 point change, $n=21 ; p=0.008)$ as well as the second procedure (12.2 point change, $n=23 ; p=$ 0.002). In addition, significant improvements in bodily pain (9.9 point change, $\mathrm{n}=21 ; p=0.047)$ after the first procedure were observed.

HRQOL is increasingly being used as an important prognostic tool in palliative oncology, and some have
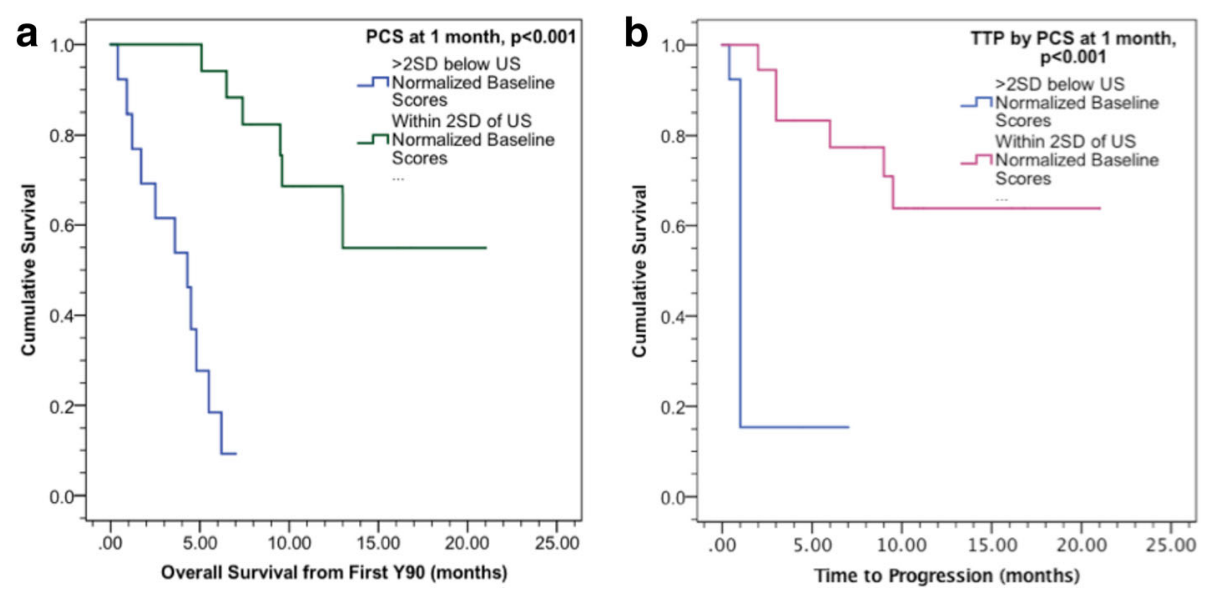

Fig. 3 Kaplan Meier analysis of HRQOL for patients who had PCS within 2 standard deviations (SD) vs. those with PCS $>2 S D$ below US normalized baseline scores. At 1 month post-Y90, patients with PCS demonstrated: (a) Significantly prolonged median OS (15.7 months vs. 3.7 months; $p<0.001)$; and (b) Significantly prolonged time to progression (12.4 months vs. 1.8 months; $p<0.001)$ compared those with physical component scores $>2$ SD below normalized US population values 


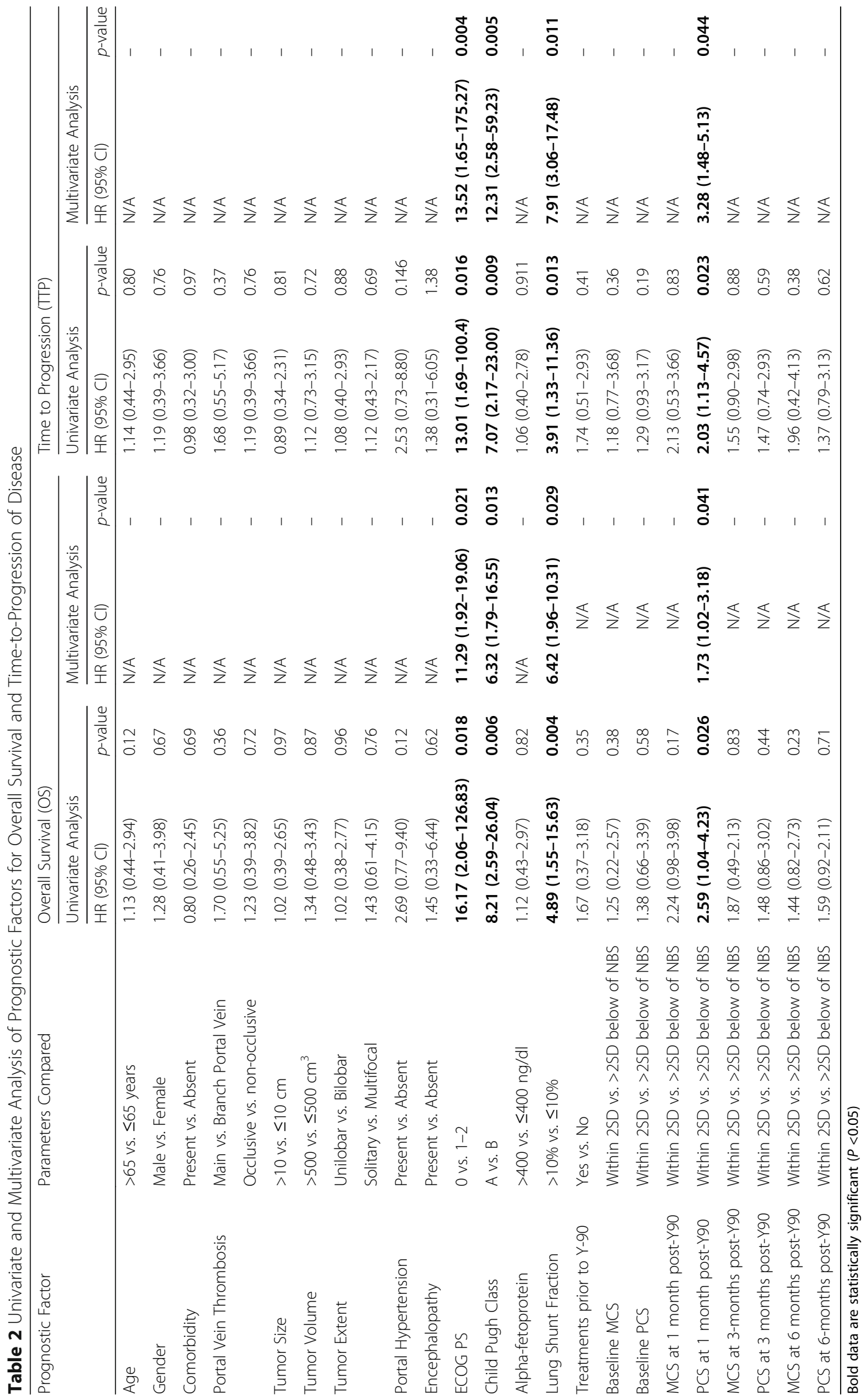


reported that it may be just as useful as objective outcomes such as survival or tumor response [2, 27, 28]. When examining patients with chronic liver disease in the presence or absence of HCC, Kondo et al. found that even though HCC patients had worse physical and overall HRQOL, it was liver function and not the presence of HCC that served as main determinant of HRQOL [24, 29, 30]. Our study demonstrated positive trends in HRQOL, particularly in the Role-Emotional, Role-Physical, Vitality and Social Functioning domains. Concurrently, there were no significant decreases in any of the remaining domains at 1-3-, or 6-months following treatment. This suggests that it is possible to treat with advanced, infiltrative HCC with PVT with ${ }^{90} \mathrm{Y}$ radioembolization and increase survival without significant concern about further decreases in HRQOL in these patients.

Our study also demonstrated a median OS of 13.0 months from initial ${ }^{90} \mathrm{Y}$ radioembolization and a median time to progression of 9.0 months. The survival outcomes of ${ }^{90} \mathrm{Y}$ radioembolization for infiltrative $\mathrm{HCC}$ with PVT have not been widely reported. However, given that all patients in this study were BCLC Stage C, such survival times are comparable with other published studies assessing the efficacy of DEB-TACE in HCC, with median OS of 13.3 months [31] to 13.5 months [32] in BCLC Stage C patients alone. Prior studies of noninfiltrative HCC patients with PVT reported median OS of 10 to 13 months [33, 34]. In our study, patients with PCS within 2SD of NBS demonstrated significantly increased median OS (15.7 months vs. 3.7 months, $p<$ 0.001 ) and significantly prolonged time to progression (12.4 months vs. 1.8 months, $p<0.001$ ) compared those with PCS $>2$ SD below a healthy, age-adjusted US population. This was the case despite the fact that patients with PCS within 2SD of NBS had significantly higher mean index tumor size $(9.7 \mathrm{~cm}$ vs. $9.1 \mathrm{~cm}, p=0.04)$ compared to patients with PCS $>2$ SD below normalized US population. Furthermore, PCS at 1 -month post ${ }^{90} \mathrm{Y}$ radioembolization was a significant independent prognostic indicator for prolonged overall survival (HR 1.73; 95\% CI $1.02-3.18 ; p=0.041)$ and time to progression (HR 3.28; 95\% CI 1.48-5.13; $p=0.044$ ), and significant decreases in PCS were independent predictors of poorer OS and TTP. In addition to the highlighting the value of physical component assessments in predicting patient survival, these results indicate the potentially integral role HRQOL analysis may play in comparative effectiveness analysis of therapeutic options.

The strengths of this study included its prospective nature as a correlative study with a phase II randomized controlled trial, with extensive short- and medium-term follow-up of patient outcomes in advanced, infiltrative HCC with PVT. Some limitations included the study's small sample size, the fact that not all patients were able to complete surveys at all follow-up intervals, due either to worsening clinical status or death and subsequent loss to follow-up. In addition, as all HRQOL survey measures are dependent on multifactorial internal and external influences, possible bias (including reporter and observer bias) and confounding factors cannot be completely ruled out.

\section{Conclusions}

In summary, our study demonstrated that health-related quality of life was preserved in the short- and mediumterm for patients who received ${ }^{90} \mathrm{Y}$ radioembolization for infiltrative, advanced HCC with PVT. Furthermore, ECOG performance status, Child-Pugh score, lung shunt fraction and 1-month post $-{ }^{90} \mathrm{Y}$ therapy physical component summary scores were independent prognostic factors for overall survival from therapy.

\section{Abbreviations}

BCLC: Barcelona Clinic Liver Cancer; CLIP: Cancer of the Italian Liver Programme; cTACE: Conventional transarterial chemoembolization; DEBTACE: Drug eluting bead transarterial chemoembolization;

HCC: Hepatocellular Carcinoma; HRQOL: Health-related quality of life; MELD: Model for End-Stage Liver Disease; PVT: Portal vein thrombosis; SF36: Short-Form 36; UNOS: United Network for Organ Sharing; Y90: Yttrium-90 Radioembolization

\section{Acknowledgements \\ None}

Funding

Not applicable.

\section{Availability of data and materials}

The data that support the findings of this study were generated at Emory University School of Medicine and may be available upon reasonable request with permission from that institution. As all authors of this study are no longer affiliated with Emory University School of Medicine, the authors are unable to make these data publicly available.

\section{Authors' contributions}

MX conducted analysis and interpretation of the data, statistical analysis, drafting of the manuscript, and critical revision of the manuscript for important intellectual content. NK was involved in acquisition of data; analysis and interpretation of data; drafting of the manuscript; critical revision of the manuscript for important intellectual content; and statistical analysis. JCC was involved in acquisition of data; analysis and interpretation of data; and critical revision of the manuscript for important intellectual content. HSK was involved in study concept and design; acquisition of data; analysis and interpretation of data; drafting of the manuscript; critical revision of the manuscript for important intellectual content; statistical analysis; and study overall supervision. All authors were involved in critically revising the manuscript prior to final submission. All authors read and approved the final manuscript.

\section{Ethics approval and consent to participate}

Ethics approval to conduct this study was provided by the Emory University Institutional Review Board. Informed consent to participate in this study was obtained from each participant prior to study enrollment. The study was registered at ClinicalTrials.gov, identifier NCT01556282, on March 16, 2012

\section{Consent for publication}

This manuscript contains only aggregate results and does not present any individual person's data. This manuscript does not contain details, images, or videos relating to any individual person and therefore does not require written informed consent from each individual for publication. 


\section{Competing interests}

The authors declare that they have no competing interests.

\section{Publisher's Note}

Springer Nature remains neutral with regard to jurisdictional claims in published maps and institutional affiliations.

\section{Author details}

${ }^{1}$ Interventional Radiology, Department of Radiology and Biomedical Imaging, Yale University School of Medicine, New Haven, CT, USA. ${ }^{2}$ Interventional Radiology, Department of Radiology, the Medical University of South Caroline, Charleston, SC, USA. ${ }^{3}$ Yale Cancer Center, Yale University School of Medicine, 330 Cedar Street, TE 2-224, New Haven, CT 06510, USA.

Received: 2 October 2016 Accepted: 14 December 2017

Published online: 12 January 2018

\section{References}

1. Bosch FX, Ribes J, Cleries R, Diaz M. Epidemiology of hepatocellular carcinoma. Clin Liver Dis. 2005;9(2):191-211.

2. Toro A, Pulvirenti E, Palermo F, Di Carlo I. Health-related quality of life in patients with hepatocellular carcinoma after hepatic resection, transcatheter arterial chemoembolization, radiofrequency ablation or no treatment. Surg Oncol. 2012;21(1):e23-30.

3. Jemal A, Bray F, Center MM, Ferlay J, Ward E, Forman D. Global cancer statistics. CA Cancer J Clin. 2011;61(2):69-90.

4. Cheung TK, Lai CL, Wong BC, Fung J, Yuen MF. Clinical features, biochemical parameters, and virological profiles of patients with hepatocellular carcinoma in Hong Kong. Aliment Pharmacol Ther. 2006; 24(4):573-83.

5. Llovet JM, Bustamante J, Castells A, Vilana R, Ayuso Mdel C, Sala M, Bru C, Rodes J, Bruix J. Natural history of untreated nonsurgical hepatocellular carcinoma: rationale for the design and evaluation of therapeutic trials. Hepatology. 1999;29(1):62-7.

6. Llovet JM, Bru C, Bruix J. Prognosis of hepatocellular carcinoma: the BCLC staging classification. Semin Liver Dis. 1999;19(3):329-38.

7. Yeung YP, Lo CM, Liu CL, Wong BC, Fan ST, Wong J. Natural history of untreated nonsurgical hepatocellular carcinoma. Am J Gastroenterol. 2005; 100(9):1995-2004

8. Schoniger-Hekele M, Muller C, Kutilek M, Oesterreicher C, Ferenci P, Gangl A Hepatocellular carcinoma in Central Europe: prognostic features and survival. Gut. 2001;48(1):103-9.

9. Jang ES, Yoon JH, Chung JW, Cho EJ, SJ Y, Lee JH, Kim YJ, Lee HS, Kim CY. Survival of infiltrative hepatocellular carcinoma patients with preserved hepatic function after treatment with transarterial chemoembolization. J Cancer Res Clin Oncol. 2013;139(4):635-43.

10. Jelic S, Sotiropoulos GC, Group EGW. Hepatocellular carcinoma: ESMO clinical practice guidelines for diagnosis, treatment and follow-up. Ann Oncol. 2010;21(Suppl 5):v59-64.

11. Lau WY, Sangro B, Chen PJ, Cheng SQ, Chow P, Lee RC, Leung T, Han KH, Poon RT. Treatment for hepatocellular carcinoma with portal vein tumor thrombosis: the emerging role for radioembolization using yttrium-90. Oncology. 2013;84(5):311-8.

12. Chung GE, Lee JH, Kim HY, Hwang SY, Kim JS, Chung JW, Yoon JH, Lee HS, Kim YJ. Transarterial chemoembolization can be safely performed in patients with hepatocellular carcinoma invading the main portal vein and may improve the overall survival. Radiology. 2011;258(2):627-34.

13. Luo J, Guo RP, Lai EC, Zhang YJ, Lau WY, Chen MS, Shi M. Transarterial chemoembolization for unresectable hepatocellular carcinoma with portal vein tumor thrombosis: a prospective comparative study. Ann Surg Oncol. 2011;18(2):413-20

14. Lopez RR, Jr., Pan SH, Hoffman AL, Ramirez C, Rojter SE, Ramos H, McMonigle M, Lois J: Comparison of transarterial chemoembolization in patients with unresectable, diffuse vs focal hepatocellular carcinoma. Archives of surgery (Chicago, III : 1960) 2002, 137(6):653-657; discussion 657-658.

15. Salem R, Lewandowski RJ, Mulcahy MF, Riaz A, Ryu RK, Ibrahim S, Atassi B, Baker T, Gates V, Miller FH, et al. Radioembolization for hepatocellular carcinoma using Yttrium-90 microspheres: a comprehensive report of longterm outcomes. Gastroenterology. 2010;138(1):52-64.
16. Kokabi N, Camacho JC, Xing M, El-Rayes BF, Spivey JR, Knechtle SJ, Kim HS Open-label prospective study of the safety and efficacy of glass-based yttrium 90 radioembolization for infiltrative hepatocellular carcinoma with portal vein thrombosis. Cancer. 2015;

17. Bonnetain F, Paoletti X, Collette S, Doffoel M, Bouche O, Raoul $J$, Rougier $P$, Masskouri F, Barbare JC, Bedenne L. Quality of life as a prognostic factor of overall survival in patients with advanced hepatocellular carcinoma: results from two French clinical trials. Qual Life Res. 2008;17(6):831-43.

18. Diouf M, Filleron T, Barbare JC, Fin L, Picard C, Bouche O, Dahan L, Paoletti $X$, Bonnetain $F$. The added value of quality of life (QoL) for prognosis of overall survival in patients with palliative hepatocellular carcinoma. J Hepatol. 2013;58(3):509-21

19. Ware JEJ, Sherbourne CD. The MOS 36-item short-form health survey (SF-36).।. Conceptual framework and item selection. Med Care. 1992;30(6):473-83.

20. Wible BC, Rilling WS, Drescher P, Hieb RA, Saeian K, Frangakis C, Chen Y Eastwood D, Kim HS. Longitudinal quality of life assessment of patients with hepatocellular carcinoma after primary transarterial chemoembolization. J Vasc Interv Radiol. 2010;21(7):1024-30.

21. Salem R, Gilbertsen M, Butt Z, Memon K, Vouche M, Hickey R, Baker T, Abecassis MM, Atassi R, Riaz A, et al. Increased quality of life among hepatocellular carcinoma patients treated with radioembolization, compared with chemoembolization. Clin Gastroenterol Hepatol. 2013;11(10) 1358-1365.e1351

22. Kanematsu M, Semelka RC, Leonardou P, Mastropasqua M, Lee JK. Hepatocellular carcinoma of diffuse type: MR imaging findings and clinica manifestations. J Magn Reson Imaging. 2003:18(2):189-95.

23. Kokabi N, Camacho JC, Xing M, El-Rayes BF, Spivey JR, Knechtle SJ, Kim HS Open-label prospective study of the safety and efficacy of glass-based yttrium 90 radioembolization for infiltrative hepatocellular carcinoma with portal vein thrombosis. Cancer. 2015;121(13):2164-74.

24. Steel J, Baum A, Carr B. Quality of life in patients diagnosed with primary hepatocellular carcinoma: hepatic arterial infusion of cisplatin versus 90yttrium microspheres (Therasphere ${ }^{\circledast}$ ). Psycho-Oncology. 2004;13(2):73-9.

25. Steel $J$ L. Clinically meaningful changes in health-related quality of life in patients diagnosed with hepatobiliary carcinoma. Ann Oncol. 2006; 17(2):304-12

26. Sun V, Ferrell B, Juarez G, Wagman LD, Yen Y, Chung V. Symptom concerns and quality of life in hepatobiliary cancers. Oncol Nurs Forum. 2008;35(3):E45-52.

27. Fan SY, Eiser C, Ho MC. Health-Related Quality of Life in Patients With Hepatocellular Carcinoma: A Systematic Review. Clinical Gastroenterology and Hepatology. 2010;8(7):559-564.e510

28. DeSalvo KB, Bloser N, Reynolds K, He J, Muntner P. Mortality prediction with a single general self-rated health question. A meta-analysis. J Gen Intern Med. 2006;21(3):267-75.

29. Kondo Y, Yoshida H, Tateishi R, Shiina S, Mine N, Yamashiki N, Sato S, Kato $N$, Kanai F, Yanase $M$, et al. Health-related quality of life of chronic liver disease patients with and without hepatocellular carcinoma. J Gastroenterol Hepatol. 2007;22(2):197-203.

30. Bianchi G, Loguercio C, Sgarbi D, Abbiati R, Brunetti N, De Simone T, Zoli M, Marchesini G. Reduced quality of life of patients with hepatocellular carcinoma. Dig Liver Dis. 2003;35(1):46-54.

31. Kalva SP, Pectasides M, Liu R, Rachamreddy N, Surakanti S, Yeddula K, Ganguli S, Wicky S, Blaszkowsky LS, Zhu AX. Safety and effectiveness of chemoembolization with drug-eluting beads for advanced-stage hepatocellular carcinoma. Cardiovasc Intervent Radiol. 2014;37(2):381-7.

32. Prajapati HJ, Dhanasekaran R, El-Rayes BF, Kauh JS, Maithel SK, Chen Z, Kim HS. Safety and efficacy of doxorubicin drug-eluting bead transarterial chemoembolization in patients with advanced hepatocellular carcinoma. J Vasc Interv Radiol. 2013;24(3):307-15.

33. Hilgard P, Hamami M, Fouly AE, Scherag A, Muller S, Ertle J, Heusner T, Cicinnati VR, Paul A, Bockisch A, et al. Radioembolization with yttrium-90 glass microspheres in hepatocellular carcinoma: European experience on safety and long-term survival. Hepatology. 2010;52(5):1741-9.

34. Mazzaferro V, Sposito C, Bhoori S, Romito R, Chiesa C, Morosi C, Maccauro M, Marchiano A, Bongini M, Lanocita R, et al. Yttrium-90 radioembolization for intermediate-advanced hepatocellular carcinoma: a phase 2 study. Hepatology. 2013;57(5):1826-37. 